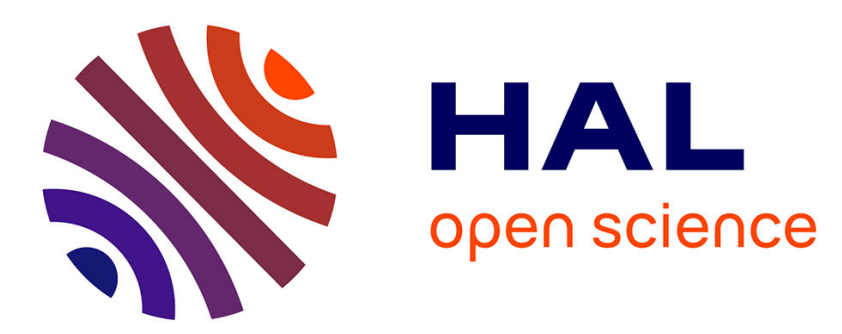

\title{
Large-Scale MIMO Receiver Based on Finite-Alphabet Sparse Detection and Concave-Convex Optimization
}

\author{
Yacine Meslem, Abdeldjalil Aissa El Bey, Mustapha Djeddou
}

\section{To cite this version:}

Yacine Meslem, Abdeldjalil Aissa El Bey, Mustapha Djeddou. Large-Scale MIMO Receiver Based on Finite-Alphabet Sparse Detection and Concave-Convex Optimization. IEEE International Workshop on Signal Processing Advances in Wireless Communications (SPAWC), May 2020, Atlanta, United States. 10.1109/SPAWC48557.2020.9154216 . hal-02861823

\section{HAL Id: hal-02861823 \\ https://imt-atlantique.hal.science/hal-02861823}

Submitted on 9 Jun 2020

HAL is a multi-disciplinary open access archive for the deposit and dissemination of scientific research documents, whether they are published or not. The documents may come from teaching and research institutions in France or abroad, or from public or private research centers.
L'archive ouverte pluridisciplinaire HAL, est destinée au dépôt et à la diffusion de documents scientifiques de niveau recherche, publiés ou non, émanant des établissements d'enseignement et de recherche français ou étrangers, des laboratoires publics ou privés. 


\section{Large-Scale MIMO Receiver Based on Finite-Alphabet Sparse Detection and Concave-Convex Optimization}

\author{
Yacine Meslem \\ Laboratoire Télécommunications, \\ École Militaire Polytechnique, \\ B.P 17, Bordj el Bahri (16111), Algeria \\ yacinemeslem@hotmail.fr
}

\author{
Abdeldjalil Aïssa-El-Bey \\ IMT Atlantique, \\ UMR CNRS 6285 Lab-STICC, \\ Brest F-29238, France \\ abdeldjalil.aissaelbey@imt-atlantique.fr
}

\author{
Mustapha Djeddou \\ Laboratoire Télécommunications, \\ École Militaire Polytechnique, \\ B.P 17, Bordj el Bahri (16111), Algeria \\ djeddou.mustapha@gmail.com
}

\begin{abstract}
In this paper, we propose a new receiver for detecting signals in large-scale Spatially Multiplexed (SP) MultipleInput-Multiple-Output (MIMO) systems that may have fewer receive antennas than transmitted symbols (overloaded case). Relying on the idea of Finite-Alphabet Sparse (FAS) detection, we formulate the Maximum Likelihood (ML) criterion as a Difference-of-Convex (DC) programming problem that can be simply and efficiently solved using the Concave-Convex Procedure (CCP) technique. Since, the considered problem is nonconvex, we theoretically discuss the behavior of the derived algorithm. Numerical experiments confirm the superiority of the proposed detection scheme, when compared with recent detection methods based on convex optimization, in a variety of large-scale MIMO transmission scenarios including the overloaded case.

Index Terms-Large-scale MIMO, Concave-convex optimization, Finite-alphabet signals, Sparse representation.
\end{abstract}

\section{INTRODUCTION}

A detector that enjoys both sufficient accuracy and lowcomputational cost is fundamental for achieving the great features of large-scale (massive) Multiple-Input-Multiple-Output (MIMO) systems [1]. These features, namely in terms of channel capacity and high data rates are required for the fifth generation of mobile networks (5G) [2]. Because of the large system dimensions, the Maximum Likelihood (ML) detector is computationally unrealizable. Moreover, classical approaches applicable to conventional MIMO systems (with few antennas) cannot be exploited in large-scale ones; either for the growth in computational complexity or for the severe degradation in performance. At one extreme, linear detector like the Minimum Mean Square Error (MMSE) are attractive from a computational-complexity point of view, but their Bit Error Rate (BER) performance is far from being optimal. At the other extreme, sphere decoder-based algorithms provide an excellent BER at the cost of a burden computational complexity [3].

Receivers, for Spatially Multiplexed (SP) large-scale MIMO systems are usually designed under the assumption that the number of receive antennas is at least equal to the number of the transmit symbols. This assumption, however, cannot be fulfilled in some interesting situations. For example, when a
Non-Orthogonal Multiple Access (NOMA) scheme is adopted in up-link, the number of antennas $N_{r}$ in the base station may be fewer than the number of users' terminals times their number of antennas $N_{t}$.

Essentially motivated by the above, new detection schemes that borrow compressive sensing (CS) [4] models based on convex optimization have been recently proposed [5]-[9]. Interestingly, these detection schemes have shown an attractive tradeoff between complexity and performance, together with a remarkable efficiency in handling the overloaded case i.e., when $N_{r}<N_{t}$. For instance, the Iterative Weighted Sum of Absolute Values (IW-SOAV) detector [5] is built upon the convex Sum of Absolute Values (SOAV) method for the reconstruction of sparse finite-alphabet vectors from incomplete linear measurements [10]. Thereby, at each iteration, IW-SOAV updates the weights and solves a weighted SOAV problem. It has been later extended to the so-called Iterative Weighted Sum of Sparse Regularizers (IW-SCSR) to directly handle complex-valued signals [6]. It worth mentioning that the success, of both IW-SOAV and IW-SCSR, depends on several parameters, which require a careful tuning along with the detection problem instance. This may partially explain why the use of these detectors for higher-order QAM signals has not been yet investigated.

Contrary to IW-SOAV and IW-SCSR, detection schemes presented in [7]-[9] are non-iterative, in the sense that, they only require the solution of one convex optimization problem. Detectors in [7], [8] are based on a convex relaxation of an exact reformulation of the ML criterion obtained by adopting the idea of Finite-Alphabet Sparse (FAS) detection introduced in [11]. Similarly, the authors in [9], have used the simplicity principle, in CS, to propose another convex criterion for detecting signals in large scale MIMO systems.

In order to take more advantage of the ML reformulation presented in [7], [8], we propose in this work, a new largescale SP-MIMO receiver based on a non-convex criterion. More precisely, we formulate the detection problem as a (smooth) Difference-of-Convex (DC) programming [12] problem. Then, we resort on the Concave Convex Procedure (CCP) 
[13] to propose a simple and efficient algorithm. As we shall see, this strategy results naturally in an iterative detection scheme like IW-SOAV and IW-SCSR.

The rest of this paper proceeds as follows. In Section II, we describe our data model. In Section III, we review the FAS detection approach. We give a detailed derivation of our detection scheme in Section IV. The examination of the proposed detector through numerical experiments is presented in Section V. Finally, we conclude this paper in Section VI.

Useful Notation: As a widely adopted convention, we use uppercase boldface letters for matrices and lowercase boldface letters for vectors. The $i^{\text {th }}$ standard unit vector of a Euclidean space $\mathbb{R}^{n}$ is denoted as $\mathbf{e}_{i}$. We denote the $d \times d$ identity matrix by $\mathbf{I}_{d}$ and the all-ones vector of length $d$ by $\mathbf{1}_{d}$. The symbols $(\cdot)^{T}$ and $(\cdot)^{H}$ stand for the transpose and the Hermitian of a matrix(resp. vector), respectively. As usual, $\Re\{\cdot\}$ and $\Im\{\cdot\}$ are used for the real part and the imaginary part of a complex number (resp. vector), while $|\cdot|$ is applied element-wise (for a vector) and denote the absolute value. We use $\operatorname{diag}(\mathbf{x})$ to design diagonal matrices constructed with the entries of a vector $\mathbf{x}$. By the symbol $\otimes$, we denote the Kronecker product. We denote the gradient of a smooth function and the subgradient of non-differentiable function $f$ at a point $\mathbf{x} \in \mathbb{R}^{n}$ as $\nabla f(\mathbf{x})$ and $\tilde{\nabla} f(\mathbf{x})$, respectively. We denote, the vector norms $\ell_{1}$ and $\ell_{2}$ by $\|\cdot\|_{1}$ and $\|\cdot\|_{2}$, respectively, while the norm-like function $\ell_{0}$ is denoted as $\|\cdot\|_{0}$.

\section{DATA MODEL}

We interest in large-scale SP-MIMO systems where, $N_{t}$ different symbols are simultaneously transmitted using $N_{t}$ antennas and received by $N_{r}$ receive antennas. We allow that $N_{r}<N_{t}$, and we call the large-scale MIMO system in this case overloaded or underdetermined. The communication is done throughput a fading channel which is represented by a matrix $\mathbf{H} \in \mathbb{C}^{N_{r} \times N_{t}}$ whose entries are independent and identically distributed (i.i.d) standard complex Gaussian variables. The observed vector $\mathbf{y}$, at the receiver, reads

$$
\mathbf{y}=\mathbf{H x}+\boldsymbol{\eta},
$$

where, $\mathrm{x} \in \mathcal{M}^{N_{t}}$ and $\mathcal{M}$ is the signaling set. The vector $\boldsymbol{\eta} \in \mathbb{C}^{N_{r}}$ represents the receiver noise and it follows a complex multivariate Gaussian distribution with $\mathbf{0}$ mean and $\sigma^{2} \mathbf{I}_{N_{r}}$ covariance matrix. We further assume that no precoding scheme is used, and that the Channel State Information (CSI) is completely available at the receiver.

Since the elements of $\mathbf{x}$ are discrete, the optimal ML detector maps to an integer least square problem given by

$$
\begin{array}{ll}
\text { minimize } & \|\mathbf{y}-\mathbf{H x}\|_{2}^{2} \\
\text { subject to } & \mathbf{x} \in \mathcal{M}^{N_{t}} .
\end{array}
$$

It is well known that (2) is an NP-hard problem and thus, there is no known polynomial-time algorithm which can solve it exactly.

\section{Finite-Alphabet Sparse Detection}

Before introducing our detection scheme, we review, in this section, the FAS detection (transform-based) approach [11]. The core idea of FAS relies on a sparse decomposition of the dense vector $\mathbf{x}$ of the transmitted data. To be concrete, let us consider a symbol $m_{i} \in \mathcal{M}=\left\{m_{1}, \ldots, m_{L}\right\}$. Using the fact that $\mathcal{M}$ is finite, one can decompose $m_{i}$ as

$$
m_{i}=\mathbf{m}^{T} \mathbf{e}_{i} .
$$

Here, $\mathbf{m}=\left[m_{1}, \cdots, m_{L}\right]^{T}$ and $\mathbf{e}_{i} \in \mathbb{R}^{L}$ is the sparse representation of $m_{i}$. It is straightforward to construct a dictionary that sparsely represent $\mathrm{x}$ such that [7], [11]

$$
\mathbf{x}=\left(\mathbf{I}_{N_{t}} \otimes \mathbf{m}^{T}\right) \tilde{\mathbf{s}}=\tilde{\mathbf{D}} \tilde{\mathbf{s}}
$$

where, $\tilde{\mathbf{s}} \in \mathbb{R}^{L N_{t}}$ is the sparse decomposition of $\mathbf{x}$ and $\tilde{\mathbf{D}}$ is the dictionary. Note that, the size of $\tilde{\mathbf{s}}$ is $L$ times higher than that of $\mathbf{x}$ and hence, this decomposition is not so attractive for higher-order alphabets [8]. For that, the so-called half sparse decomposition has been proposed [8]. This representation is performed in two steps. First, the QAM symbols are mapped to BPSK points [14]. Then, the resulting BPSK symbols are sparsely decomposed using (3). The size of the sparse representation is now reduced to $2 p N_{t}$, where, $p=\log _{2}(L)$.

If the half-sparse representation is considered, the ML detector has been proved to be equivalent to [7], [8]

$$
\begin{array}{ll}
\operatorname{minimize} & \|\mathbf{y}-\mathbf{\Phi} \mathbf{s}\|_{2}^{2} \\
\text { subject to } & \mathbf{B s}=\mathbf{1}_{p N_{t}} \\
& \|\mathbf{s}\|_{0}=p N_{t} .
\end{array}
$$

The matrix $\mathbf{\Phi}=\mathbf{H D}$, where, $\mathbf{D}$ is now the dictionary and $\mathbf{s}$ is the half-sparse representation of $\mathbf{x}$. The matrix $\mathbf{B}=\mathbf{I}_{p N_{t}} \otimes \mathbf{1}_{2}$.

Problem (4) can be thought of as a sparse recovery problem for which, a rich set of approaches has been developed in the last two decades. For instance, the convex relaxation approach based on substituting the non-convex $\ell_{0}$ function for its convex envelop ( $\ell_{1}$ norm) is popular. This approach has been already used in [7] and [8] to formulate the largescale MIMO detection problem as a convex problem, which can be exactly and efficiently solved by any available convex optimization solver, such as the interior point [7], [8] or the Alternating Direction Method of Multipliers (ADMM) method [15]. Despite the good performance of these (convex) detectors, and the effectiveness of the convex relaxation approach in solving sparse recovery problems, a better method, however, is to rely on non-convex techniques. This can be motivated, for example, by the Reweighted $\ell_{1}$ (RL1) approach, which is known to outperform the convex $\ell_{1}$ method [16].

\section{Proposed Detector Based on Concave-Convex OPTIMIZATION}

The RL1 method is based on approximating the norm-like $\ell_{0}$ with a certain smooth concave function [16], that promotes more sparsity than the $\ell_{1}$ norm. The resulting problem (after approximation) can be solved with general optimization methods such as Majorization-Minimization (MM) algorithms [16]. With this in mind, we derive, hereafter, our detector for large-scale (possibly overloaded) MIMO systems. 


\section{A. Reformulation and approximation}

Problem (4) can be rewritten, in a more convenient form, as follows

$$
\begin{array}{ll}
\text { minimize } & \|\mathbf{y}-\mathbf{\Phi} \mathbf{s}\|_{2}^{2}+\tau\|\mathbf{s}\|_{0} \\
\text { subject to } & \mathbf{B s}=\mathbf{1}_{p N_{t}} .
\end{array}
$$

Note that, for some carefully chosen penalty parameter $\tau>0$, problem (4) and (5) are equivalent.

One of the smooth concave functions that can well approximate the $\ell_{0}$ function is given by

$$
h(\mathbf{s})=\frac{2}{\pi} \sum_{i=1}^{2 p N_{t}} \arctan \left(\frac{\left|s_{i}\right|}{\epsilon}\right),
$$

where, $\epsilon>0$ is a small number controlling the approximation accuracy and $\frac{2}{\pi}$ serves as a normalization factor. Now, by substituting $\|\mathbf{s}\|_{0}$ for $h(\mathbf{s})$, problem (5) can be converted to the following minimization

$$
\begin{array}{ll}
\text { minimize } & \|\mathbf{y}-\mathbf{\Phi} \boldsymbol{s}\|_{2}^{2}+\tau h(\mathbf{s}) \\
\text { subject to } & \mathbf{B s}=\mathbf{1}_{p N_{t}} .
\end{array}
$$

Clearly, problem (7) fits into the class of DC programming problems [12]. For solving such problems, the ConcaveConvex Procedure (CCP) [13] is a well suitable MM method.

\section{B. Proposed Algorithm}

$\mathrm{CCP}$ is an iterative procedure that solves a DC problem by solving a series of convex problems. At iteration, say $k+1$, one solves a convex problem obtained through the linearization of the concave part on the neighborhood of the solution at iteration $k$ [13].

The function $h(\mathbf{s})$ can be majorized around a point $\mathbf{u}$, thanks to its concavity, as follows

$$
h(\mathbf{s}) \leq h(\mathbf{u})+\nabla h(|\mathbf{u}|)^{T}(\mathbf{s}-\mathbf{u}) .
$$

By using the majorization (8), it is not hard to see that, at the $(k+1)^{t h}$ iteration, we solve the following convex problem

$$
\begin{array}{ll}
\operatorname{minimize} & \|\mathbf{y}-\mathbf{\Phi} \mathbf{s}\|_{2}^{2}+\tau g_{k+1}(\mathbf{s}) \\
\text { subject to } & \mathbf{B s}=\mathbf{1}_{p N_{t}} .
\end{array}
$$

Here, $g_{k+1}(\mathbf{s})=\nabla h\left(\left|\mathbf{s}^{k}\right|\right)^{T} \mathbf{s}$. It is obtained by discarding the terms of the left-hand side in (8) that do not depend on the optimization variable $\mathbf{s}$. A simple calculation shows that

$$
g_{k+1}(\mathbf{s})=\left\|\mathbf{W}^{k} \mathbf{s}\right\|_{1},
$$

where, $\mathbf{W}^{k}=\operatorname{diag}\left(\left[w_{1}^{k}, \ldots, w_{2 p N_{t}}^{k}\right]\right)$ is called the weights matrix and $w_{i}^{k}=\frac{\epsilon^{2}}{\epsilon^{2}+\left(s_{i}^{k}\right)^{2}}, \forall i=1,2, \ldots, 2 p N_{t}$.

The proposed detector is detailed in Algorithm 1. It can be regarded as a series of convex detectors weighted by $\mathbf{W}^{k}$. It is similar to IW-SOAV and IW-SCSR but with much simpler and more natural way to update the weights.

It worth noting that, since Algorithm 1 is built upon CCP, it is only a heuristic for (7). Thus, its convergence to a global minimum, if it does, is not guaranteed. For that, we give the proposition below in order to ensure that our algorithm is a descent, or more precisely, a non-increasing algorithm.

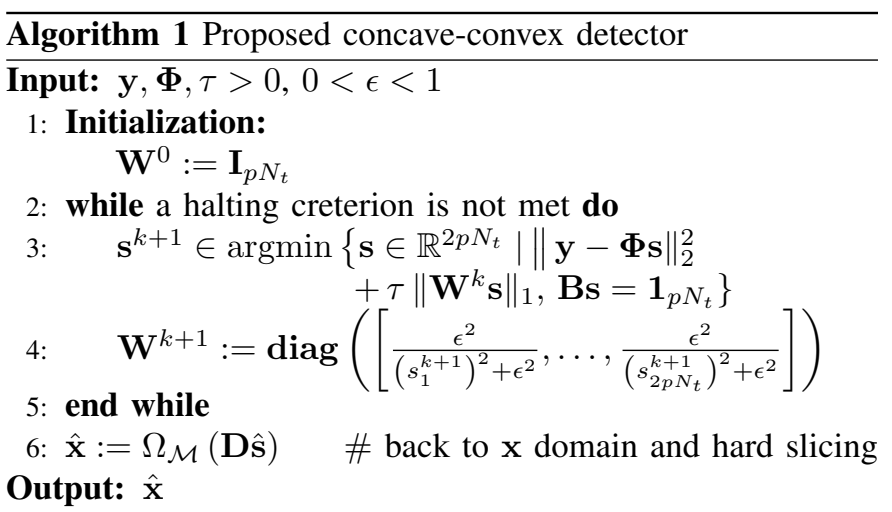

This means that it can get better approximated solution as it proceeds.

Proposition 1. Let the function $F(\mathbf{s})$ denotes the objective function in (7), i.e., $F(\mathbf{s})=\|y-\mathbf{\Phi s}\|_{2}^{2}+\tau h(\mathbf{s})$, and let $\left\{\mathbf{s}^{k}\right\}_{i=1}^{\infty}$ be the sequence generated by Algorithm 1. Then, we have

$$
F\left(\mathbf{s}^{k}\right)-F\left(\mathbf{s}^{k+1}\right) \geq 0 .
$$

Proof. For the ease of notation throughout the sketch of this proof, let $f(\mathbf{s})=\|\mathbf{y}-\mathbf{\Phi} \mathbf{s}\|_{2}$.

We begin with a basic fact about the convexity of $-h(\mathbf{s})$ (resp. the concavity of $h(\mathbf{s})$ ) which allows us to write

$$
\tau h\left(\mathbf{s}^{k}\right)-\tau h\left(\mathbf{s}^{k+1}\right) \geq-\tau \nabla h\left(|\mathbf{s}|^{k}\right)^{T}\left(\mathbf{s}^{k+1}-\mathbf{s}^{k}\right)
$$

By adding $f\left(\mathbf{s}^{k}\right)-f\left(\mathbf{s}^{k+1}\right)$ to both sides of the inequality (11) and by the definition of $g_{k+1}$ (s) (eq. (10) above), we can lower-bound $F\left(\mathbf{s}^{k}\right)-F\left(\mathbf{s}^{k+1}\right)$ in the following way

$$
\begin{aligned}
F\left(\mathbf{s}^{k}\right)-F\left(\mathbf{s}^{k+1}\right) & \geq f\left(\mathbf{s}^{k}\right)-f\left(\mathbf{s}^{k+1}\right) \\
& +\tau g_{k+1}\left(\mathbf{s}^{k}\right)-\tau g_{k+1}\left(\mathbf{s}^{k+1}\right) .
\end{aligned}
$$

Being the solution of the convex problem $(9)$ at the $(k+1)^{\text {th }}$ iteration, $\mathbf{s}^{k+1}$ satisfies the first-order optimality conditions which read

$$
\left\{\begin{array}{l}
\nabla f\left(\mathbf{s}^{k+1}\right)+\tau \tilde{\nabla} g_{k+1}\left(\mathbf{s}^{k+1}\right)+\mathbf{B}^{T} \boldsymbol{\mu}=\mathbf{0} \\
\mathbf{B s}^{k+1}=\mathbf{1}_{N_{t}},
\end{array}\right.
$$

where, $\boldsymbol{\mu} \in \mathbb{R}^{p N_{t}}$ is the Lagrange multiplier associated with the equality constraint. Left multiplying the first line in (13) by $\left(\mathbf{s}^{k+1}-\mathbf{s}^{k}\right)^{T}$ and using the fact that the scalar product is commutative for real-valued vectors, we obtain

$$
\begin{aligned}
& 0=-\nabla f\left(\mathbf{s}^{k+1}\right)^{T}\left(\mathbf{s}^{k}-\mathbf{s}^{k+1}\right) \\
& -\tau \tilde{\nabla} g_{k+1}\left(\mathbf{s}^{k+1}\right)^{T}\left(\mathbf{s}^{k}-\mathbf{s}^{k+1}\right)-\boldsymbol{\mu}^{T} \mathbf{B}\left(\mathbf{s}^{k}-\mathbf{s}^{k+1}\right) .
\end{aligned}
$$

By the feasibility of $\mathbf{s}^{k}$, i.e., $\mathbf{B} \mathbf{s}^{k}=\mathbf{1}_{p N_{t}}$, since it is the optimal solution of (9) at iteration $k$, (14) reduces to

$$
\begin{aligned}
& 0=-\nabla f\left(\mathbf{s}^{k+1}\right)^{T}\left(\mathbf{s}^{k}-\mathbf{s}^{k+1}\right) \\
& -\tilde{\nabla} g_{k+1}\left(\mathbf{s}^{k+1}\right)^{T}\left(\mathbf{s}^{k}-\mathbf{s}^{k+1}\right) .
\end{aligned}
$$


Now, by adding equation (15) side by side to inequality (12), we get

$$
\begin{aligned}
& F\left(\mathbf{s}^{k}\right)-F\left(\mathbf{s}^{k+1}\right) \geq \\
& f\left(\mathbf{s}^{k}\right)-f\left(\mathbf{s}^{k+1}\right)-\nabla f\left(\mathbf{s}^{k+1}\right)^{T}\left(\mathbf{s}^{k}-\mathbf{s}^{k+1}\right) \\
& +\tau g_{k+1}\left(\mathbf{s}^{k}\right)-\tau g_{k+1}\left(\mathbf{s}^{k+1}\right) \\
& -\tau \tilde{\nabla} g_{k+1}\left(\mathbf{s}^{k+1}\right)^{T}\left(\mathbf{s}^{k}-\mathbf{s}^{k+1}\right) .
\end{aligned}
$$

Since $f(\mathbf{s})$ is convex and smooth, it is always true that

$$
f\left(\mathbf{s}^{k}\right)-f\left(\mathbf{s}^{k+1}\right)-\nabla f\left(\mathbf{s}^{k+1}\right)^{T}\left(\mathbf{s}^{k}-\mathbf{s}^{k+1}\right) \geq 0,
$$

and by the definition of the subgradient, we have

$$
\begin{aligned}
& \tau g_{k+1}\left(\mathbf{s}^{k}\right)-\tau g_{k+1}\left(\mathbf{s}^{k+1}\right) \\
& -\tau \tilde{\nabla} g_{k+1}\left(\mathbf{s}^{k+1}\right)^{T}\left(\mathbf{s}^{k}-\tau \mathbf{s}^{k+1}\right) \geq 0 .
\end{aligned}
$$

Using (17) and (18), inequality (16) can be also lower-bounded as $F\left(\mathbf{s}^{k}\right)-F\left(\mathbf{s}^{k+1}\right) \geq 0$.

According to Proposition 1, the proposed algorithm is monotonically non-increasing. On the other hand, the objective function in (7) is always non-negative and hence, bounded from below. Thus, we can conclude that Algorithm 1 converges. However, this is not saying that it converges neither to a global nor to a local minimum. Nevertheless, the above proposition is important, since it guarantees that the proposed detector will perform, at the worst case, exactly as the convex detectors [7], [8], which are equivalent to the first iteration of Algorithm 1.

The computational cost of the proposed algorithm depends on how the convex problem (9) is solved. However, whatever the adopted method, Algorithm 1 will always have the same order of complexity as the convex detector in [8]. This is because, in practice, Algorithm 1 needs only few number of iterations to provide good performance. So, if an interior point method is employed as in [8], the complexity of our algorithm will roughly be $\mathcal{O}\left(p^{2} N_{t}^{2}\left(N_{t}+N_{r}\right)^{\frac{3}{2}}\right)$.

\section{COMPUTER EXPERIMENTS}

In this section, we present some simulation results illustrating the BER performance of the proposed receiver. For the sake of comparison, we consider the following detectors. MMSE, the convex detector of [8], IW-SOAV [5] and IW-SCSR [6]. In the figures, these detection schemes are designed by "MMSE", "Quad-min", "IW-SOAV" and "IWSCSR", respectively, while our detector is denoted by "proposed". The parameters of IW-SOAV and those of IW-SCSR are set exactly as stated in [5] and [6]. For example, the regularization parameter $\alpha$, for IW-SOAV, is set according to the SNR per receive antenna as $\alpha=0.01$ when the SNR is in range $[0,10] \mathrm{dB}$ and 0.1 when it is in $[12.5,20] \mathrm{dB}$, and the maximum number of iterations for the RachfordDouglas algorithm is 50 . For IW-SCSR, the considered sparse regularizer is $h_{2}(\mathbf{x})=\|\Re(\mathbf{x})\|_{1}+\|\Im(\mathbf{x})\|_{1}$ [6], and the ADMM algorithm iterates for at most 100 iterations. The parameter $\beta$ [6, eq. (37)] is set to 15 . We run both IW-SOAV and IW-SCSR for 5 rounds, since this gives the best of their

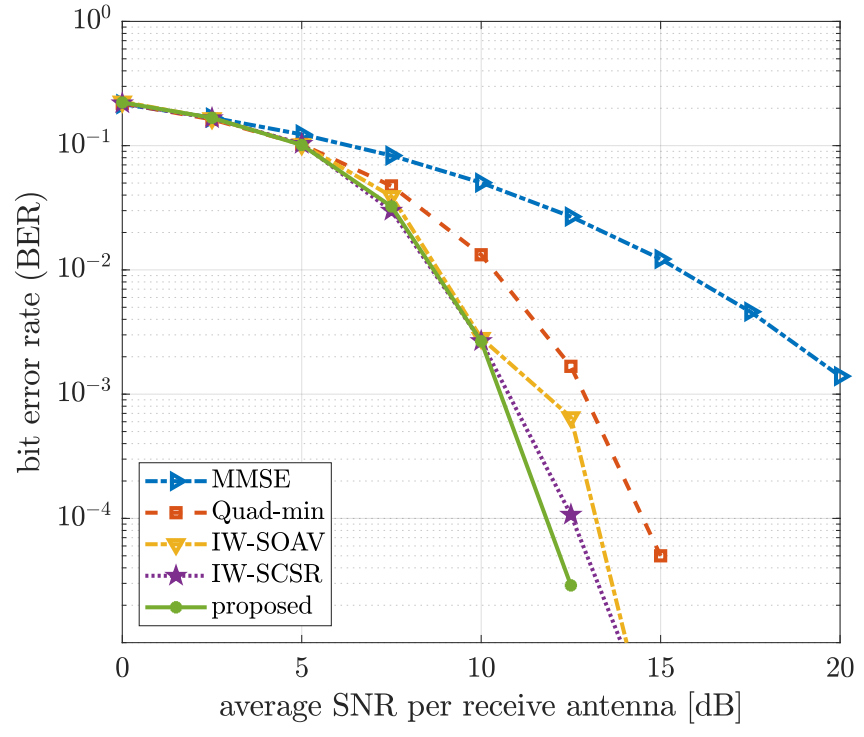

Fig. 1. BER performance of $128 \times 128$ MIMO system with QPSK

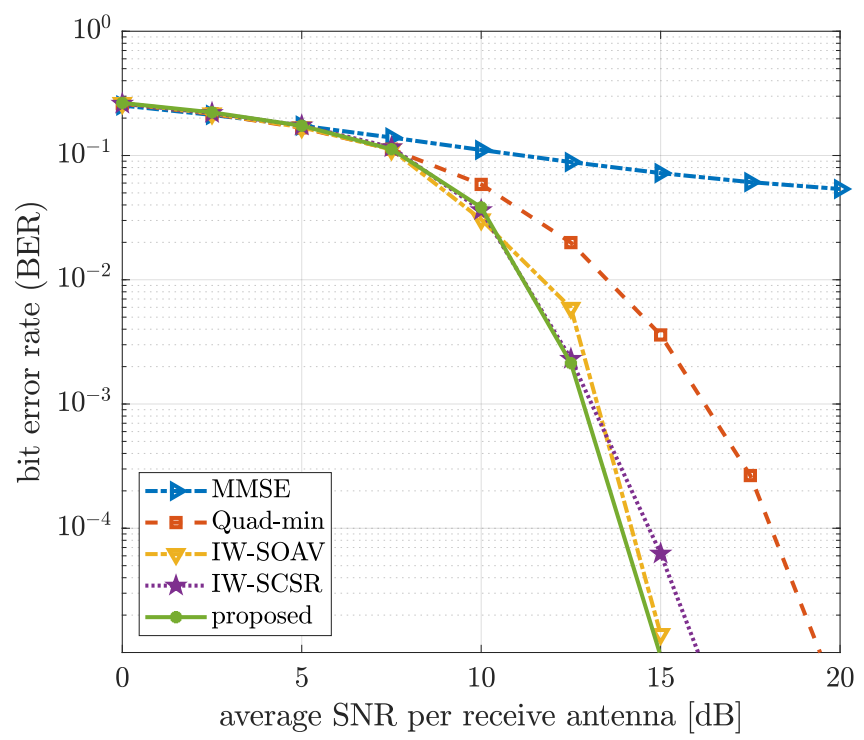

Fig. 2. BER performance of $128 \times 96$ MIMO system with QPSK

performance [5], [6]. The convex relaxation-based detector is solved with the help of the cvx package [17], which we also use for solving the series of convex problems required in our detection scheme. For all experiments, Algorithm 1 is run exactly for 3 iterations.

Fig. 1 and 2 show the BER of a determined $(128 \times 128)$ and an overloaded $(128 \times 96)$ large-scale MIMO systems, respectively, transmitting QPSK signals. The parameters of our detector, for this modulation set, are $\tau=17$ and $\epsilon=0.1$. As it can be seen, the proposed detector is superior than all other detection schemes. At BER $=10^{-4}$, it gains almost $3 \mathrm{~dB}$ in the determined case and $4 \mathrm{~dB}$ in the overloaded scenario, with respect to the convex detector. This confirm the descent nature of Algorithm 1 (Proposition 1). Observe that, IW-SOAV and IW-SCSR have very close performance. This is expected, 


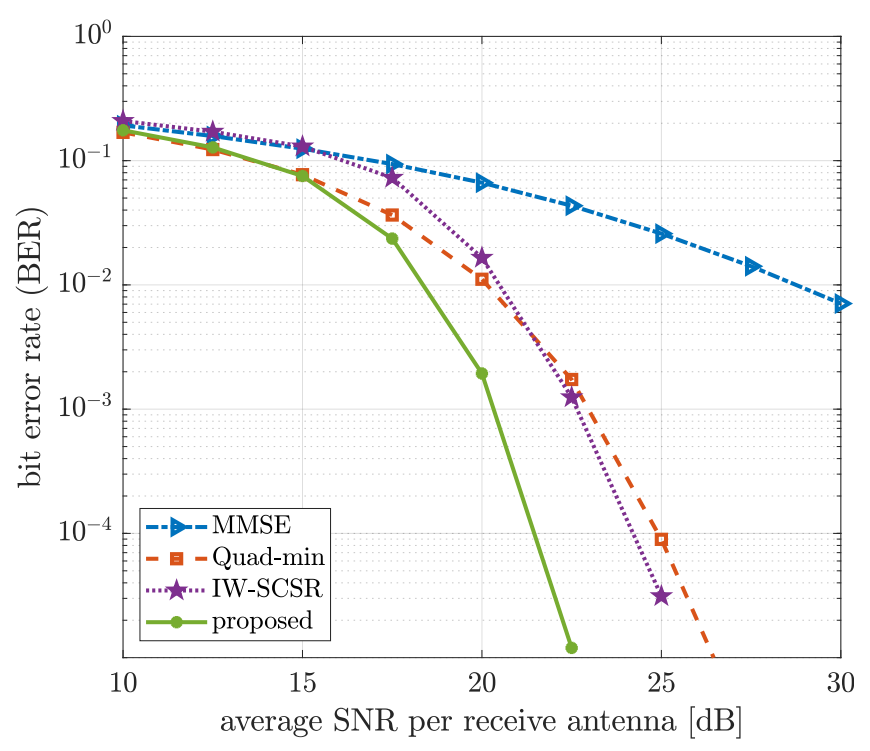

Fig. 3. BER performance of $96 \times 96$ MIMO system with $16-$ QAM

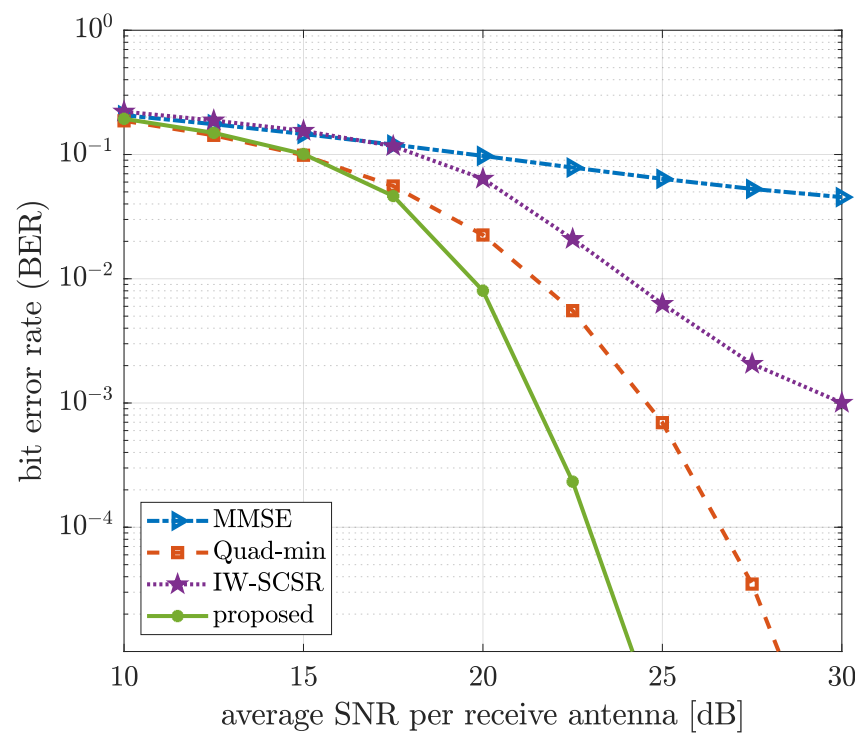

Fig. 4. BER performance of $96 \times 90$ MIMO system with $16-$ QAM

because they are equivalent for complex-valued symbols like QPSK [6]. The unexpected behavior of IW-SOAV in some SNR points, for the $128 \times 128$ configuration, may be due to its parameters, which seem to require a tuning every-time the transmission settings change.

Fig. 3 and 4 show the BER for 16-QAM modulation of a $96 \times 96$ and a $96 \times 90$ MIMO system, respectively. IWSOAV is excluded from the comparison, since, beyond QPSK alphabet, its parameters are not available. The parameters of the proposed detector for 16-QAM are $\tau=170$ and $\epsilon=0.01$. Again, the proposed detector shows the best BER performance either for the determined (Fig. 3) or the overloaded case (Fig. 4), with a gain, for example, of about $4 \mathrm{~dB}$ at a BER of $10^{-4}$, when compared to the convex detector. Note that, IW-SCSR performs poorly for this signaling scheme.

\section{CONCLUSION}

We have proposed a concave-convex criterion for detecting SP signals in large-scale MIMO systems. We have also developed a simple and efficient algorithm based on CCP. The proposed detection scheme is guaranteed to perform, at least, as a convex relaxation-based detector. As a future work, we want to investigate the impact of the parameters $\tau$ and $\epsilon$ on the performance of our detector. Moreover, it will be important to provide a stronger statement on the convergence of the proposed algorithm. The use of simpler and more tailored methods such as ADMM, for the solution of the convex problems arising in our detection scheme, will certainly help in further decreasing the computational cost.

\section{REFERENCES}

[1] R. C. de Lamare, "Massive MIMO systems: Signal processing challenges and future trends," URSI Radio Science Bulletin, vol. 2013, no. 347, pp. 8-20, Dec 2013.

[2] E. G. Larsson, O. Edfors, F. Tufvesson, and T. L. Marzetta, "Massive MIMO for next generation wireless systems," IEEE Communications Magazine, vol. 52, no. 2, pp. 186-195, February 2014.

[3] B. Hassibi and H. Vikalo, "On the sphere-decoding algorithm I. expected complexity,' IEEE Transactions on Signal Processing, vol. 53, no. 8, pp. 2806-2818, Aug 2005.

[4] D. L. Donoho, "Compressed sensing," IEEE Transactions on Information Theory, vol. 52, no. 4, pp. 1289-1306, April 2006.

[5] R. Hayakawa and K. Hayashi, "Convex optimization-based signal detection for massive overloaded mimo systems," IEEE Transactions on Wireless Communications, vol. 16, no. 11, pp. 7080-7091, Nov 2017.

[6] — , "Reconstruction of complex discrete-valued vector via convex optimization with sparse regularizers," IEEE Access, vol. 6, pp. 66499 $66512,2018$.

[7] Y. Fadlallah, A. Aïssa-El-Bey, K. Amis, D. Pastor, and R. Pyndiah, "New iterative detector of MIMO transmission using sparse decomposition," IEEE Transactions on Vehicular Technology, vol. 64, no. 8, pp. 34583464, Aug 2015.

[8] Z. Hajji, K. Amis, A. Aïssa-El-Bey, and F. Abdelkefi, "Low-complexity half-sparse decomposition-based detection for massive MIMO transmission," in 2015 5th International Conference on Communications and Networking (COMNET), Nov 2015, pp. 1-6.

[9] Z. Hajji, A. Aïssa-El-Bey, and K. Amis, "Simplicity-based recovery of finite-alphabet signals for large-scale MIMO systems," Digital Signal Processing, vol. 80, pp. 70 - 82, 2018.

[10] M. Nagahara, "Discrete signal reconstruction by sum of absolute values," IEEE Signal Processing Letters, vol. 22, no. 10, pp. 1575-1579, Oct 2015.

[11] A. Aïssa-El-Bey, D. Pastor, S. M. A. Sbaï, and Y. Fadlallah, "Sparsitybased recovery of finite alphabet solutions to underdetermined linear systems," IEEE Transactions on Information Theory, vol. 61, no. 4, pp. 2008-2018, April 2015.

[12] L. T. H. An and P. D. Tao, "The DC (difference of convex functions) programming and DCA revisited with DC models of real world nonconvex optimization problems," Annals of Operations Research, vol. 133, no. 1 , pp. 23-46, Jan 2005.

[13] A. L. Yuille and A. Rangarajan, "The concave-convex procedure," Neural Computation, vol. 15, no. 4, pp. 915-936, 2003.

[14] Jinho Choi, "Iterative receivers with bit-level cancellation and detection for MIMO-BICM systems," IEEE Transactions on Signal Processing, vol. 53, no. 12, pp. 4568-4577, Dec 2005.

[15] Y. Meslem, A. Aïssa-El-Bey, and M. Djeddou, "A simple ADMM solution to sparse-modeling-based detectors for massive MIMO systems," in 1st Conference on Electrical Engineering, Alger, Algeria, Apr. 2019. [Online]. Available: https://hal.archives-ouvertes.fr/hal02087659

[16] E. J. Candès, M. B. Wakin, and S. P. Boyd, "Enhancing sparsity by reweighted $\ell_{1}$ minimization," Journal of Fourier Analysis and Applications, vol. 14, no. 5, pp. 877-905, Dec 2008.

[17] M. Grant and S. Boyd, "CVX: Matlab software for disciplined convex programming, version 2.1," http://cvxr.com/cvx, Mar. 2014. 